\title{
Experimental study on the formation of subaqueous barchan dunes in closed conduits
}

\author{
Carlos A. Alvarez ${ }^{*}$, and Erick Franklin ${ }^{1}$ \\ ${ }^{1}$ School of Mechanical Engineering - University of Campinas - UNICAMP, Rua Mendeleyev, 200 - Campinas - SP - CEP: \\ 13083-860, Brazil
}

\begin{abstract}
The present paper reports the formation of subaqueous barchan dunes by analyzing the temporal evolution of their main geometrical characteristics (width $\mathrm{W}$, length $\mathrm{L}$ and horn lengths Lh). After certain time, the dunes reach an equilibrium state and it is possible to study the relation between $\mathrm{W}$ versus $\mathrm{L}$, and the dependence of the dune velocity on L. The barchan dunes were formed from spherical glass and zirconium beads. An initial conical heap of beads was placed on the bottom wall of a rectangular channel and it was entrained by a water turbulent flow. The evolution of the dunes was filmed with a CCD camera placed above the channel and mounted on a traveling system. Our results show that after a characteristic time the dune shape does not change and it travels with a roughly constant velocity. Once the equilibrium state is reach, W and $\mathrm{L}$ are measured, showing linear dependence. Furthermore, we show that the dune velocity $\mathrm{V}_{\mathrm{d}}$ scales with the inverse of the dune length.
\end{abstract}

\section{Introduction}

The transport of solid particles entrained by a fluid flow is frequently found in both nature and industry. When shear stresses exerted by the fluid on the bed of particles are bounded to some limits, a mobile layer of particles known as bed-load takes place in which the particles stay in contact with the fixed bed. If it takes place over a nonerodible ground or with limited sediment supply from the bed, they form dunes showing a remarkable crescentic shape with horns pointing downstream [1]. These dunes, known as barchan dunes, are commonly observed in deserts. However, field measurements suffer from the lack of control on meteorology conditions as well as the large length and time scales (100 $\mathrm{m}$ and 1 year) involved in the physics of dunes [2]. The shape and size selection of dunes, their dynamics, and even their stability remain as open issues [3]. Creating dunes in a controlled way and using shorter scales could help to improve our knowledge on those questions. Similar barchan dunes have also been observed under water flows (with much shorter lengths than in air). Barchan dunes under water have received much less attention than aeolian dunes, and important issues remain unanswered. Recently, some numerical studies have been conducted to investigate the evolution of barchan dunes $[4,5]$.

Nowadays, the availability of high-performance computational tools allows the use of numerical simulations to study complex phenomena like the formation of barchan dunes. Therefore, experimental studies are vital to generate data and validate those numerical simulations. This work presents some experiments with glass and zirconium beads entrained by a turbulent water flow. Its objective is to investigate the formation and velocity of barchan dunes.

\section{Experimental device}

The experimental device consisted of a water reservoir, two centrifugal pumps, a flow straightener, a $5 \mathrm{~m}$ long channel, a settling tank, and a return line. The flow straightener was a divergent-convergent nozzle filled with $\mathrm{d}=3 \mathrm{~mm}$ glass spheres, the function of which was to homogenize the flow profile at the channel inlet. The channel had a rectangular cross-section (width $=160 \mathrm{~mm}$ and height $2 \delta=50 \mathrm{~mm}$ ) and was made of transparent material. Figure 1 shows the scheme of the experimental loop. The channel test section was $1 \mathrm{~m}$ long and started 40 hydraulic diameters $(3 \mathrm{~m})$ downstream of the channel inlet (the turbulent flow was completely developed in the test section). The particles were placed in the channel test section, which was previously filled with water. The grains settled in the water at rest and formed a conical heap. Next, a water flow was imposed in the channel, and the heap deformed into a barchan dune.

The evolution of the dune shape was recorded with a CCD camera placed above the channel and mounted on a traveling system. With this procedure, each experiment concerns one single isolated dune. In our experiments, we used water as the fluid media and the granular material was spherical beads: glass beads with density $\rho_{\mathrm{s}}=2500$ $\mathrm{kg} / \mathrm{m}^{3}$ and mean diameter $\mathrm{d}=0.37 \mathrm{~mm}$, and heavier zirconium particles with density $\rho_{\mathrm{s}}=4000 \mathrm{~kg} / \mathrm{m}^{3}$ and mean diameter $\mathrm{d}=0.50 \mathrm{~mm}$.

\footnotetext{
* Corresponding author: calvarez@fem.unicamp.br
} 
Head Tank

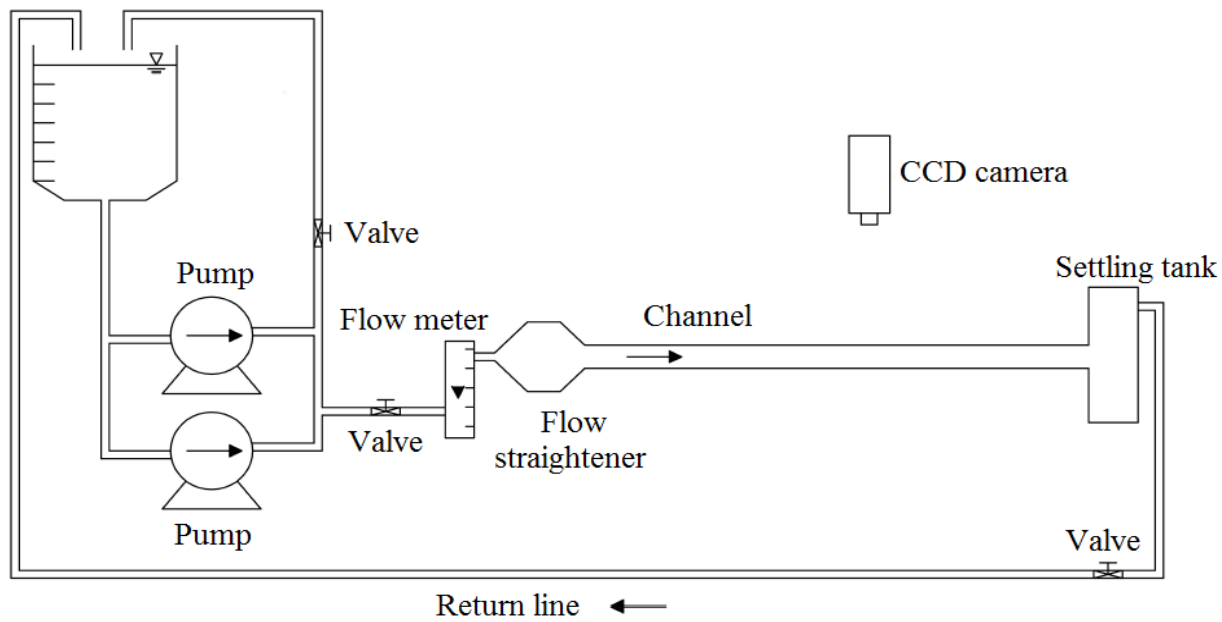

Fig. 1. Schematic of the experimental loop. (Figure extracted from the Supplemental Material of [6]).

The water flow rate was varied between 6.5 and $9.5 \mathrm{~m}^{3} / \mathrm{h}$. The flow velocity $\bar{U}$, defined as the ratio of the measured volumetric flow rate and the channel cross-section, was varied between 0.22 and $0.33 \mathrm{~m} / \mathrm{s}$. The corresponding Reynolds number, with $v$ as the kinematic viscosity, was calculated using the Eq. 1 and it was in the range $11280-$ 16700 .

$$
R e=\frac{\bar{U} 2 \delta}{v}
$$

\section{Dune morphology}

Once a conical heap of grains is formed in the channel, the flow is started up at a constant rate. Before being displaced over a measurable distance, the pile is deformed and adopts a crescentic shape as shown in Fig. 2.
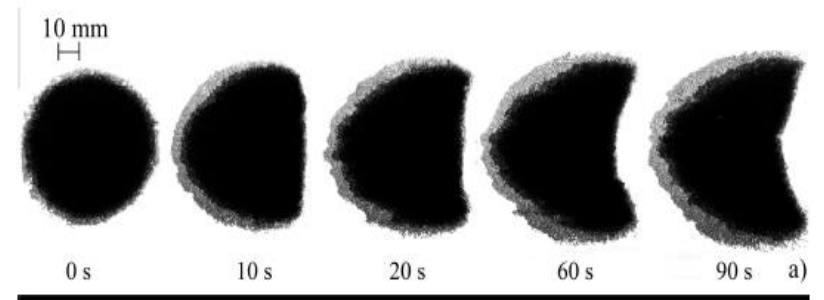

$10 \mathrm{~s}$ $20 \mathrm{~s}$ $60 \mathrm{~s}$ $90 \mathrm{~s} \quad$ a)
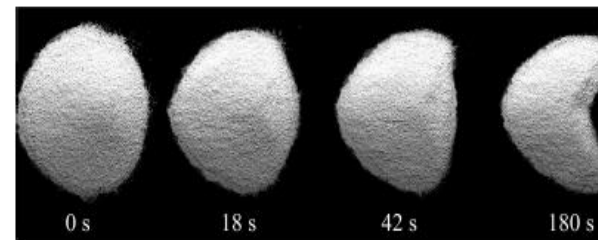

$180 \mathrm{~s}$
A schematic view of a barchan dune is shown in Fig. 3, which defines its dimensions. $\mathrm{L}$ is the distance, in the symmetry plane, from the front to the rear of the dune, not taking into consideration the horns. $\mathrm{W}$ is the dune width, $\mathrm{L}_{\mathrm{h}}$ is the horns length and $\mathrm{H}$ is the dune height. An image processing code, written as a Matlab script, was developed during this work to automatically treat the acquired images. The code tracks the dunes along the images and computes its $\mathrm{L}, \mathrm{W}, \mathrm{L}_{\mathrm{h}}$, and the centroid position; from the latter the dune velocity $\mathrm{V}_{\mathrm{d}}$ is computed.

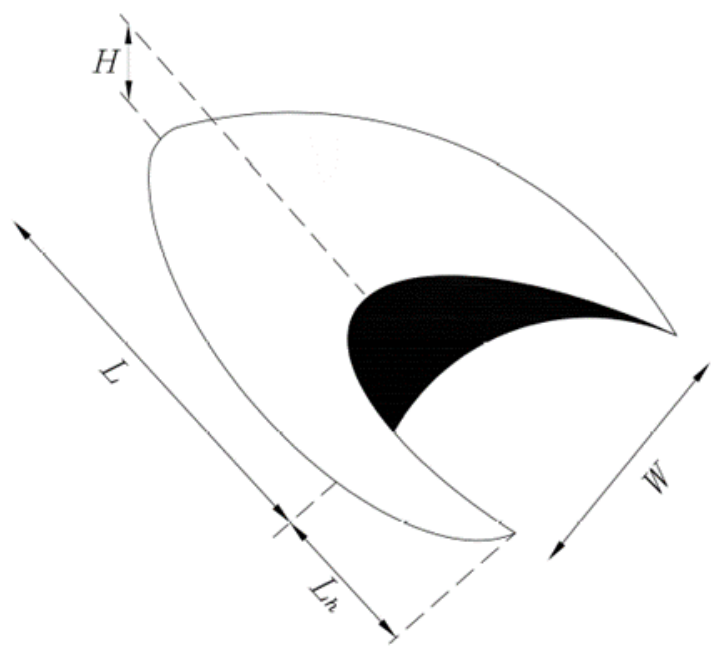

Fig. 3. Scheme of a barchan dune showing its dimensions.

\section{Results and discussion}

Fig. 2. Top views of initially conical heaps deposited on the channel test section and deformed by the water flow at different times, the water flow is from left to right. a) Black glass beads with mean diameter of $0.37 \mathrm{~mm}$ at $\mathrm{Re}=14700$. b) Zirconium beads with mean diameter of $0.50 \mathrm{~mm}$ at $\mathrm{Re}=16700$.
To analyze the formation of barchan dunes, we started by evaluating their temporal evolution. It is considered as initial state a conical heap of grains. As soon as the water flow begins, the heap deforms and to adopt a "croissant" shape. 
Our results show the evolution of some geometrical parameters of dunes along the time. Figure 4 shows the formation of barchan dunes, through $\mathrm{W}$ and $\mathrm{W} / \mathrm{L}$, considering the initial state at time equals to zero. Figure 5 shows the centroid positions along the time, the dune velocity $\mathrm{V}_{\mathrm{d}}$ can be obtained by computing the slopes in this figure. $V_{d}$ can be calculated once the dunes had reached their equilibrium shape, i.e., after they had traveled a distance of the order of the size of the initial heap [7]. For the tests displayed in Fig. 5, the equilibrium state is approximately reached at time equals to $8 \mathrm{~s}$. That time also corresponds to when the dune width $\mathrm{W}$ is roughly the same as the length L (see Fig. 4). Each continuous line in the Figs 4 and 5 corresponds to a barchan dune analyzed during a test run with $\mathrm{Re}=16500$. Asterisks represent a mean value for the observations analyzed.

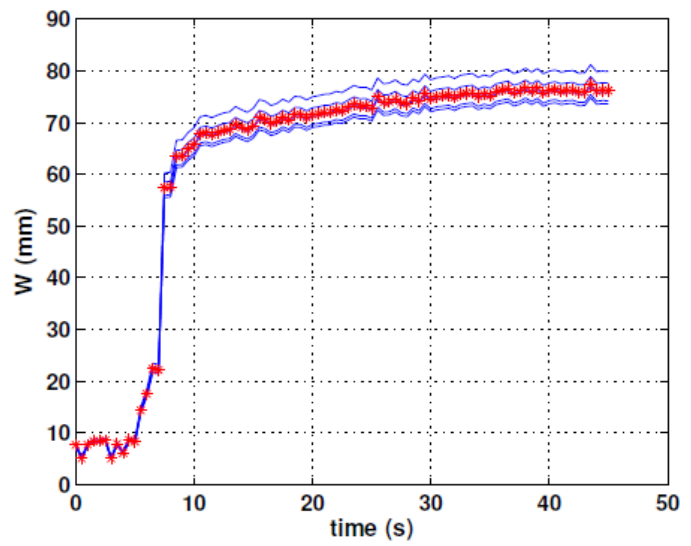

(a)

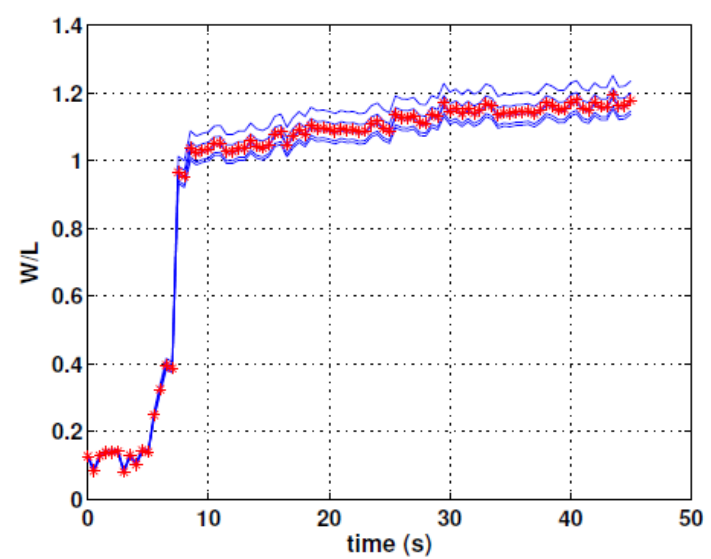

(b)

Fig. 4. (a) Evolution of dune width $\mathrm{W}$ along the time. (b) Evolution of W normalized by the dune length L. The particles employed were spherical glass beads with mean diameter of 0.37 $\mathrm{mm}, \mathrm{Re}=16700$. Each line corresponds to a different barchan dune. (*) mean value of $\mathrm{W}$ along the time

Once the equilibrium state was reached we calculated the length $\mathrm{L}$ and the width $\mathrm{W}$ of barchan dunes. This procedure was performed for a large number of test runs. Figure $6 \mathrm{a}$ and $6 \mathrm{~b}$ show the width $\mathrm{W}$ versus the length $\mathrm{L}$, respectively, for black glass beads $(\mathrm{d}=0.37 \mathrm{~mm})$ and zirconium beads $(\mathrm{d}=0.50 \mathrm{~mm})$, for four different Reynolds numbers. Each one of the symbols in these figures represents one test run. $\mathrm{W}$ is approximately related to $\mathrm{L}$ with a linear dependence, and this relationship appears to be independent of the particle diameter and density.

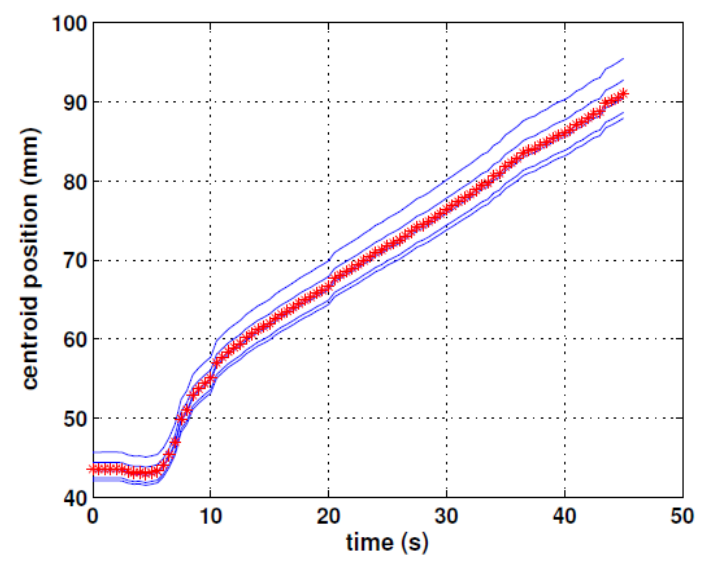

Fig. 5. Centroid position versus the time. The inclination gives the dune velocity $V_{d}, R e=16700$. Each line corresponds to a barchan dune observed in a test run. (*) mean value of observations.

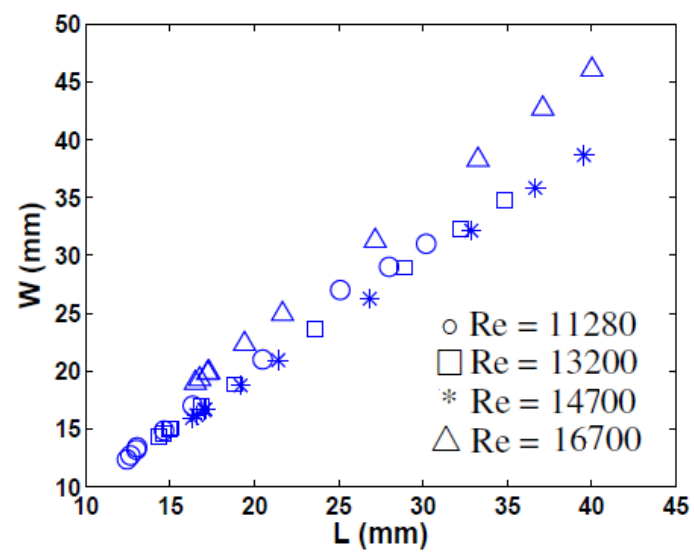

(a)

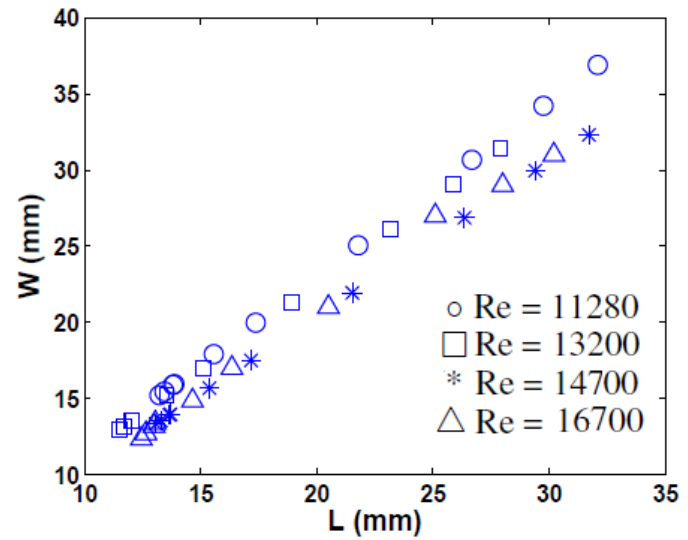

(b)

Fig. 6. (a) Width $\mathrm{W}$ versus the length $\mathrm{L}$ for four different Reynolds number. The symbols used are explained in the key. (a) Black glass beads with mean diameter $\mathrm{d}=0.37 \mathrm{~mm}$. (b) Zirconium beads with $\mathrm{d}=0.50 \mathrm{~mm}$.

Figure 7 displays the velocity $\mathrm{V}_{\mathrm{d}}$ as a function of the inverse dune length $(1 / \mathrm{L})$, for four different Reynold 
numbers, and for the two types of beads employed in this study. It is possible to identify that for a given Reynolds number, the dune velocity scales with the inverse length, $\mathrm{V}_{\mathrm{d}} \propto 1 / \mathrm{L}$. Furthermore, in Fig. 7 it is clearly visible that, given a dune length, the dune velocity increases with the Reynolds number. These results are in good agreement with previously published works $[2,8]$.

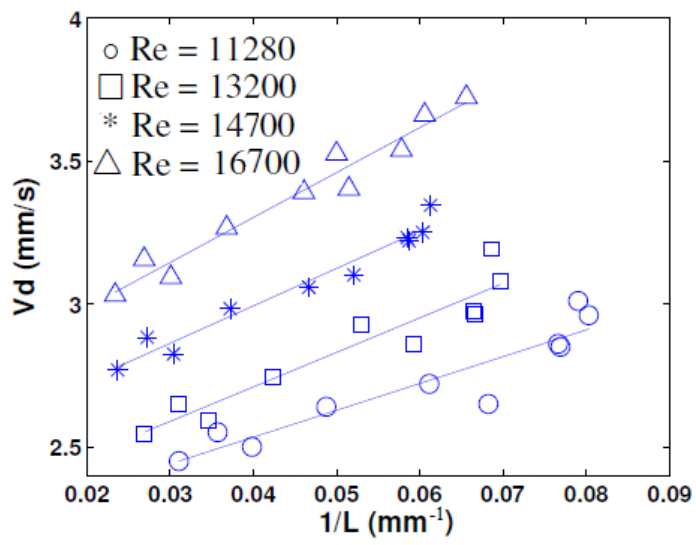

(a)

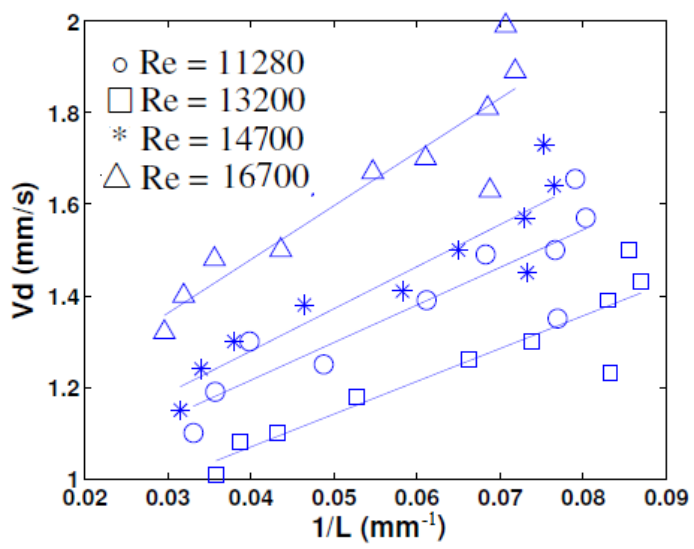

(b)

Fig. 7. Dune velocity $V_{d}$ versus the inverse length $1 / L$ for four different Reynolds number. The symbols used are explained in the key. (a) Black glass beads with mean diameter $d=0.37 \mathrm{~mm}$. (b) Zirconium beads with $\mathrm{d}=0.50 \mathrm{~mm}$. The continuous lines emphasize the linear dependence $\mathrm{V}_{\mathrm{d}} \propto 1 / \mathrm{L}$ for the given flow conditions.

\section{Conclusions}

The present paper reported the evolution of a conical heap of grains entrained by a turbulent flow that evolved to a barchan dune. The grains were formed from glass and zirconium beads. The formation of the dunes was filmed with a CCD camera. An image processing code was developed to identify the dunes, follow them along the images, and to calculate some geometrical parameters that define their morphology. Our results showed that after a characteristic time the dune shape does not change, and it travels with a roughly constant velocity.

The linear relationship between the width and length appears to be independent of the particle diameter and density. The velocity scales with the inverse of the dune length, and for the two grain types grain used the dune velocity increases with the Reynolds number for a given dune length. An important issue to future is to study the formation of barchan dunes employing grains with different characteristics, for instance, using beads with irregular shapes. Other important task is to test the evolution of barchan consisting of grains of two different specific masses.

Carlos A. Alvarez is grateful to the Ecuadorian government foundation SENESCYT for the scholarship grant (no. 2013AR2Q2850) and to CNPq (grant no. 140773/2016-9). Erick Franklin is grateful to FAPESP (grant nos. 2012/19562-6 and 2016/13474-9), to CNPq (grant no. 400284/2016-2) and to FAEPEX/UNICAMP (grant no. 2210/17)) for the financial support provided.

\section{References}

1. H.J. Herrmann, G. Sauermann, Physica A 283, 24-30 (2000)

2. P. Hersen, S. Douady, B. Andreotti, Phys. Rev. Lett. 89, No. 264301 (2002)

3. P. Hersen, K.H. Andersen, H. Elbelrhiti, B. Andreotti, P. Claudin, S. Douady, Phys. Rev. E 69, No. 011304 (2004)

4. A. Khosronejad, F. Sotiropoulos, J. Fluid Mech. 815, 117-148 (2017)

5. A.G. Kidanemariam, M. Uhlmann, J. Fluid Mech. 818, 716-743 (2017)

6. C.A. Alvarez, E. Franklin, Phys. Rev. E 96, No. 062906 (2017)

7. E.M. Franklin, F. Charru, Powder Technology 190, 247-251 (2009)

8. E.M. Franklin, F. Charru, J. Fluid Mech. 675, 199$222(2011)$ 